In Stage 0, cisternal pressure gradually increased with compensation of some extent. Venous blood flow and venous pressure decreased slowly. In Stage 1 and 2 , respiration became progressively slowly. Cisternal pressure rose and venous flow and pressure fell rapidly. Anisocoria, then bilateral mydriasis appeared. In Stage 3, respiration and venous flow ceased. Venous pressure became zero. Cisternal pressure often rapidly fell in this stage, suggesting tentorial herniation occurred. In Stage 4 animal passed into shock and died. ECG alterations during compression were also characteristic. Respiratory arrhythmia without change of ECG wave form was observed in Stage 1. Inspiratory sinus rhythm, disappearance of $\mathrm{P}$, elongation of $\mathrm{P}-\mathrm{Q}$, and complete $\mathrm{A}-\mathrm{V}$ block in Stage 2. Reciprocally conducted $\mathbf{P}$ associated with sinus rhythm in this stage suggests that impulse is sent from the A-V node. In Stage 3, giant $T$ appeared. Ventricular extrasystole suddenly changed to ventricular tachycardia.

Prompt decompression in early phase of Stage 3 produced rapid fall in pulse rate and blood pressure. Venous flow rapidly increased, and was maintained at high level for considerable time, after blood pressure had returned to the original level. It suggests long lasting vasodilatation following decompression. Venous pressure also showed marked rise on decompression. Rapid evacuation in middle phase of Stage 3, after marked tachycardia had lasted for a few minutes, also produced the same changes as above mentioned. Then, however, cisternal and blood pressure started to increase again. Several to 50 minutes after decompression, the same changes in Stage 1 to 4 appeared again. The animal passed into shock and died. This phenomenon is due to the acute brain swelling after prompt decompression. If enough amount of venous blood was drained out of the cranial cavity, decompression was not followed by this phenomenon. Following decompression in late phase of Stage 3 and 4, animal died promptly without the 2nd peak. These facts suggest that cerebral vasomotor paralysis during compression plays a predominant role in the acute brain swelling of this type.

\title{
120. Circulatory Disturbances after Cerebral Ischemia
}

\author{
Masayoshi Kowada \\ 2nd Surgical Clinic, Tohoku University
}

Little attention has been given to cerebrovascular occlusion during cerebral ischemia. Obstruction of blood vessels was demonstrated in a post-ischemic brain and survival from ischemia was prolonged approximately twice in pre-heparinized animals. Factors played parts of vascular obstruction were studied from following points of views, changes of blood itself, endotherial swelling and mechanical compression by swallen tissue. One of the most important findings was obstruc- 
tion by blood itself and fibrin clot, platelet thrombus or sludging phenomenon were studied in details.

\title{
121. Electronmicroscopic Studies on Cerebral Edema, with Special Reference to Edema on the Human Brain
}

\author{
Seigo KaMaE, Tokuro Kusunoki and Takao Mitsuno \\ Department of Surgery, Kobe University School of Medicine
}

The recent advances in the field of electronmicroscopic studies on cerebral edema have been centered largely on the experimental examinations performed on animals. Reports of investigation on the clinical cases have been rather scarce

We have studied the cortex and white matter after incision of the dura, in 26 specimens taken from the neighboring tissues of the brain tumor and six specimens from cases with traumatic contusion and cerebral hematoma.

The electronmicroscopic findings were made from the panoramic picture magnified to 10,000 times the normal tissue.

Neuroglia and process of nerve cells in the cortex and subcortical white matter surrounding the brain tumor were somewhat swollen in comparison to that of the normal state. The intracellular space in the cortex was very narrow and adjacent to each other. There were no difference in the size of the extracellular space between the capillary surroundings and the peripheral area. In the cortical capillaries, the basal membrane retains its felt-like structure with partial coarsening. The process of pericapillary astrocytes were markedly enlarged, with a tendency for mitochondrial congregation appearing lateral to the basal membrane.

A marked enlargement of the extracellular space was noted in the deep white matter, and edematous picture of neuroglia cell process and a group of the myelinated fibers were seen.

In the white matter of the brain with highly advanced edema, the expanded extracellular space contained debris of the glia cells undergoing inhibition and destruction, pieces of cell membrane and swollen mitochondria. The capillary vessels are silhouetted in the extracellular space surrounded by cellular processes.

Cells, 4.5-5.5 in diameter, with oval nuclei and with or without nucleoli were found scattered around the structureless myelin. The amount of cytoplasm was rather scarce, in comparison with the nucleus.

Macrophages were composed of polysome, lamellar rough surfaced endoplasmic reticulum, vacuoles and phagosomes resembling macrophages.

In the traumatic type of cerebral contusions, clustered red cells were noted 